\title{
Usefulness of Tomographic Versus Planar Lung Scintigraphy in Suspected Pulmonary Embolism in a Daily Practice
}

\author{
Pierre Weinmann ${ }^{*}, 1$, Jean-Luc Moretti ${ }^{1}$ and Michel W. Brauner ${ }^{2}$ \\ ${ }^{I}$ Department of Nuclear Medicine, Hopital Avicenne APHP, Université Paris 13, France \\ ${ }^{2}$ Department of Radiology, Hopital Avicenne APHP, Université Paris 13, France
}

\begin{abstract}
Objectives: Lung scintigraphy is non-diagnostic in most patients suspected of pulmonary embolism when performed in planar mode. Tomographic mode could improve lung scan performance but has not been rigorously assessed. In this study we assessed the usefulness of tomographic mode in patients with a non-diagnostic lung scan scintigraphy.

Methods: pulmonary embolism was diagnosed or ruled out in consecutive patients with a non-diagnostic lung scan by the combination of a 4-slices computerized tomography and a lower-limb ultrasonography. Results given by reading the tomographic mode in ventilation/perfusion lung scan were compared to final diagnosis.

Results: 142 out of 392 consecutive patients with a non-diagnostic planar lung scintigraphy were included while 47 were excluded, 45 because of contra-indication to contrast medium and 2 because of non-optimal opacification of pulmonary arteries. Ninety-five patients were evaluated. Pulmonary embolism was diagnosed in $20(21 \%)$ patients and ruled out in 75 (79\%). Concordance with final diagnosis was found in 77/94 (82\%) patients. Tomographic mode was non-diagnostic in 1 (1\%) patient. Tomographic mode sensitivity/specificity/accuracy were $0.79 / 0.83 / 0.80$ respectively. Negative predictive value was 0.94 . Discordances were related to single sub segmental or non-occluding segmental thrombus.

Conclusion: Tomographic mode is diagnostic in nearly all patients and agreement with final diagnosis is found in the majority. Its excellent negative predictive value enables to rule out pulmonary embolism. Discordances were related to single sub segmental or non-occluding segmental thrombus which prognosis value remains to be established.
\end{abstract}

\section{INTRODUCTION}

The new Pioped recommendations for the diagnosis of pulmonary embolism have relegated ventilation/perfusion $(\mathrm{V} / \mathrm{Q})$ scintigraphy as the first imaging test to a minority of situations [1]. Patients with severe iodine allergy, impaired renal function, pregnant women or women of reproductive age represent the minority of patients in whom pulmonary scintigraphy is still indicated. A careful analysis of the recommendations of the Pioped II investigators shows however that pulmonary scintigraphy may be useful in patients with discordant findings between pre-test clinical probability assessment and computerized tomography (CT) or in patients with thrombus at the sub segmental level only.

Although CT scan has hold its promises, morbidity induced by iodinated contrast media and breast radiation in women are a concern. The vast majority of patients (a mean of $75 \%$ ) who undergo a CT scan for suspicion of PE have no PE [2-9], most are women [10] and breast radiation during CT scan is far superior to the absorbed dose with lung scintigraphy (20-50 mGy versus $0.3 \mathrm{mGy}$ ) [10-12]. This greatly exceeds the American College of Radiology recommendations of $<3 \mathrm{mGy}$ for standard two-view mammography. Breast radiation delivered by CT scan approaches or exceeds levels known to increase the probability of cancer $[12,13])$.

*Address correspondence to this author at the Department of Nuclear Medicine, Hopital Avicenne APHP, Université Paris 13, France;

E-mail: pierre.weinmann@avc.aphp.fr
Since lung scintigraphy has still a role in PE diagnosis, with no contra-indications and side-effects and a low breast radiation, any improvement in its diagnostic performances would be welcome.

Single Photon Emission Tomography (SPECT) lung scintigraphy, i.e. lung scintigraphy in tomographic mode, has been studied by several authors [14-18], but clear evidence of its usefulness has not been established yet. In Pioped studies, lung scintigraphy was performed in planar mode. The aim of our study was to assess the usefulness of tomography in patients in whom planar lung scan performed as a first imaging test was not contributive.

\section{MATERIALS AND METHODOLOGY}

This study has been approved by the ethics committee and informed consent was obtained from all subjects.

\section{Selection of Patients}

Three hundred and ninety two consecutive patients with clinical suspicion of acute PE (less than five days since the onset of symptoms) with elevated plasma D-dimer were refered for a lung scintigraphy performed as a first-line imaging test. V/Q was performed first as an imaging test by the referring practitioners for one or several of the following reasons: normal chest X ray, lack of availability of CT in an emergency setting, knowledge of our study where V/Q had to be performed first. Patients who had already undergone CT scan were excluded. Two hundred and fifty of them had a diagnostic planar extracted lung scan, performed as described further. 
Tomographic lung scintigraphy performances were assessed in the 142 remaining patients with a non-diagnostic lung scan. Further exclusion criteria were pregnancy, age less than $18 \mathrm{y}$, absolute contra-indications to contrast medium, previous history of PE.

\section{Procedures}

V/Q lung scan, plasma D-dimer measurement, chest radiography, CT scan and lower-limb US were done systematically within $48 \mathrm{~h}$ after admission.

\section{V/Q Scintigraphy: SPECT}

The V/Q lung scan was performed exclusively by a SPECT technique using a double-head $\gamma$ camera equipped with low-energy, high resolution, parallel-hole collimators (ADAC Vertex Plus and Sopha DST XL). Ventilation scan were done after inhalation of 445 to $555 \mathrm{MBq}{ }^{99 m}$ Tc Technegas over 3-5 respiratory cycles which leads to a radiation exposure of up to $1.6 \mathrm{mSv}$. Perfusion scan was done immediately after the ventilation scan with a mean activity of 300 $\mathrm{MBq}{ }^{99 m}$ Tc labelled macro-aggregated albumin $\left({ }^{99 m} \mathrm{Tc}-\right.$ MAA) (radiation exposure $3.3 \mathrm{mSv}$ ). This protocol leads to a total radiation exposure of up to $4.9 \mathrm{mSv}$.

All patients remained in supine position throughout the examination.

A $360^{\circ}$ SPECT acquisition of the pulmonary ventilation and perfusion was performed using a $128 \times 128$ matrix.

A $180^{\circ}$ rotation per head was done in 32 steps of $30 \mathrm{~s}$ each for ventilation and $20 \mathrm{~s}$ each for perfusion studies. Accordingly, the total acquisition time for ventilation and perfusion SPECT studies was 27 minutes. No respiratory gating was performed. Reconstruction of coronal, sagital and transversal slices was done by filtered back-projection followed by two iterations with a fifth-order Butterworth filter and a cut-off frequency at 0.45 .

\section{V/Q Scintigraphy: Planar Images}

Planar images on six views were created from the SPECT datasets of the ventilation and perfusion scans. Two consecutive projections were extracted and assembled to create anterior, posterior, right and left lateral, right and left oblique posterior views. This corresponds to extracted planar images. For ethical reasons, it was unacceptable to increase by 30 minutes the examination time to perform an additional regular planar study in patients suspected of PE.

In a preliminary study, we performed in 20 patients a blind comparison between regular planar images and the views obtained as previously described. Although image quality was better in the former than the latter views, final diagnosis established by a consensus reading of 3 independent referees was identical in all patients. Higher doses of radiotracers than usual were used in this study in order to get count statistics enabling the use of extracted planar images as an alternative to regular planar views. Extraction of planar projections from the SPECT datasets is an accepted substitute for planar views $[17,19]$.

\section{Interpretation Criteria}

V/Q planar lung scan: lung scans were classified as normal, very low, low, intermediate, or high probability for PE according to the updated criteria of the PIOPED II study. Lung scans of low and intermediate probability were considered non-diagnostic.

V/Q SPECT lung scan: the images of patients with a noncontributive planar scintigraphy were separately visually interpreted by two independent experienced readers unaware of the results of CT scan and lower-limb US. In case of discordant reading, a simultaneous interpretation was performed by the two readers in order to reach a consensus.

Final result had to be sorted according one of the 3 following readings:

- $\quad$ very high probability for PE

- no PE

- non-diagnostic procedure.

\section{Very High Probability for PE}

At least one segmental or sub-segmental (more than 15\% of a segment) perfusion defect with a normal corresponding ventilation (mismatch defect) (Fig. 1).

\section{No PE}

At least one matched defect (no ventilation and no perfusion) with no corresponding opacity on chest radiography, patient with ventilation and perfusion abnormalities, with more pronounced ventilations defects, absence of ventilation and perfusion of a complete lobe,

- $\quad$ ventilation and perfusion defects extending along the expected scissures, (Fig. 2).

- bilateral and symmetrical perfusion defects at both bases with pyramidal shape,

- $\quad$ perfusion abnormalities which do not match with anatomical segments.

\section{Non-Diagnostic Procedure}

Association of a corresponding ventilation and perfusion defect with a corresponding opacity on chest radiography (triple match defect), quality of the procedure considered as too low. Since all patients had a non-diagnostic V/Q planar lung scan, no patient could have a normal lung SPECT.

\section{Multidetector-Row CT}

CT scans were performed on a 4-detector multislice CT (MSCT) system Mx 8000 (Philips Medical Systems, Cleveland, Ohio). The scanning parameters were as follows: $0.5 \mathrm{~s}$ scan time, $4 \times 1 \mathrm{~mm}$ section thickness, $120 \mathrm{kV}$ and $100 \mathrm{mAs}$. Scans were performed during full inspiration whenever possible. Non-ionic intravenous contrast medium (Omnipaque 300 , Nycomed, Princeton, NJ) was injected with a power injector at rates of $3.0 \mathrm{ml} / \mathrm{s}$ for a total volume of $100 \mathrm{ml}$. Scan delay was determined by bolus timing software.

The plasma D-dimer level was measured within the first day after admission with VIDAS D-dimer (Bio-Mérieux,), a quantitative enzyme-linked immuno-sorbent assay (ELISA). A plasma D-dimer level superior to $500 \mathrm{ng} / \mathrm{L}$ was considered as positive.

Lower-limb venous compression US consisted of examination of the iliac, common and superficial femoral, popliteal 

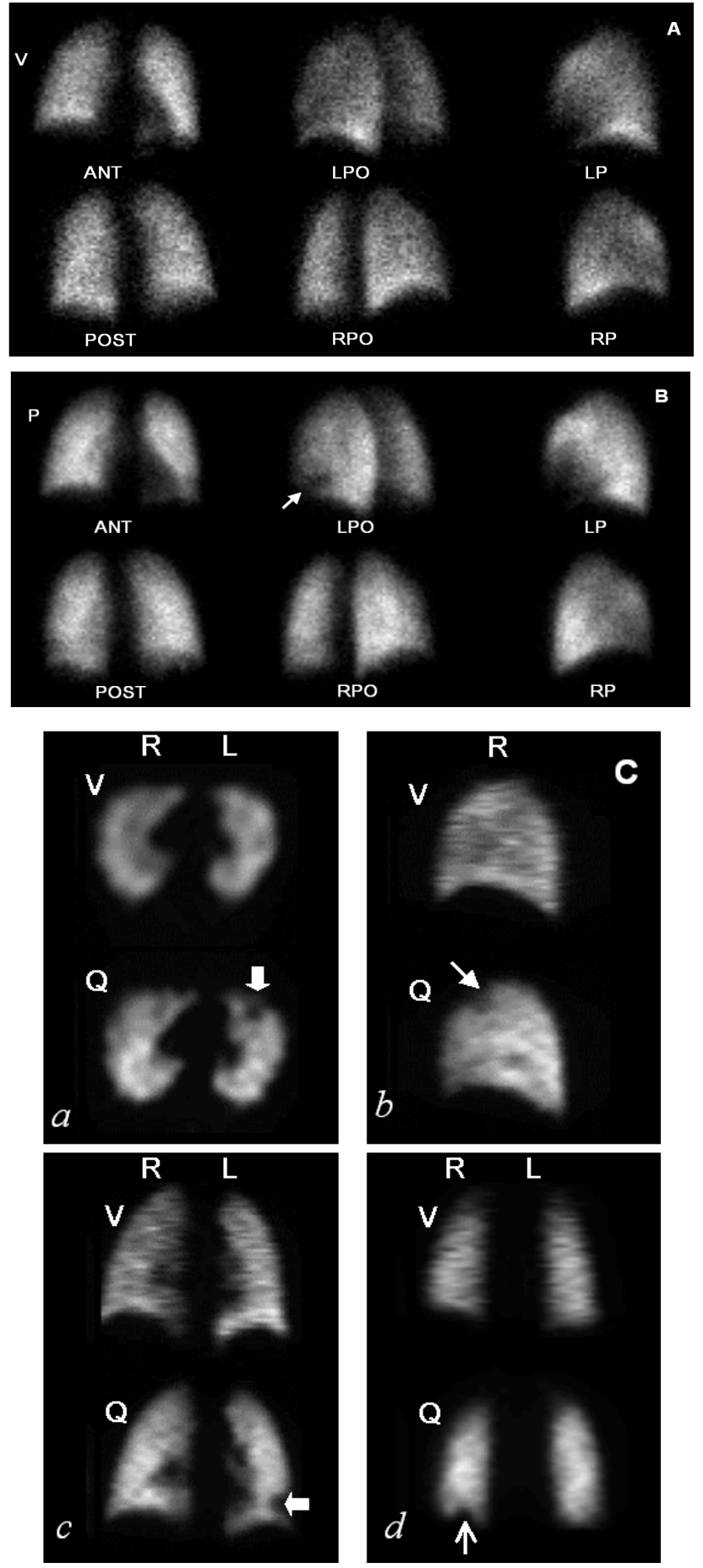

Fig. (1). Patient with bilateral sub segmental embolisms. CT scan was negative but US found a deep phlebitis. Perfusion defects are marked by arrows. Planar perfusion (B) shows a single defect in left lower lobe normaly ventilated (mismatch) on planar ventilation scintigraphy (A). Ventilation/perfusion tomography (C) reveals three subsegmental defects normaly ventilated. The triangular shape of the defects is clearly seen. The subsegmental defects in the right upper lobe (d) and the right lower lobe (c) were not visible on planar perfusion.

$\mathrm{V}=$ ventilation; $\mathrm{Q}=$ perfusion; $\mathrm{ANT}=$ anterior; $\mathrm{POST}=$ posterior; $\mathrm{RP}=$ right profile $\mathrm{LP}=$ left profile; $\mathrm{RPO}=$ right posterior oblique; $\mathrm{LPO}=$ left posterior oblique; $\mathrm{R}=$ right; $\mathrm{L}=$ left; $\mathrm{a}=$ transaxial slices; $b=$ sagittal slices of right lung; $c$ and $d=$ coronal slices.
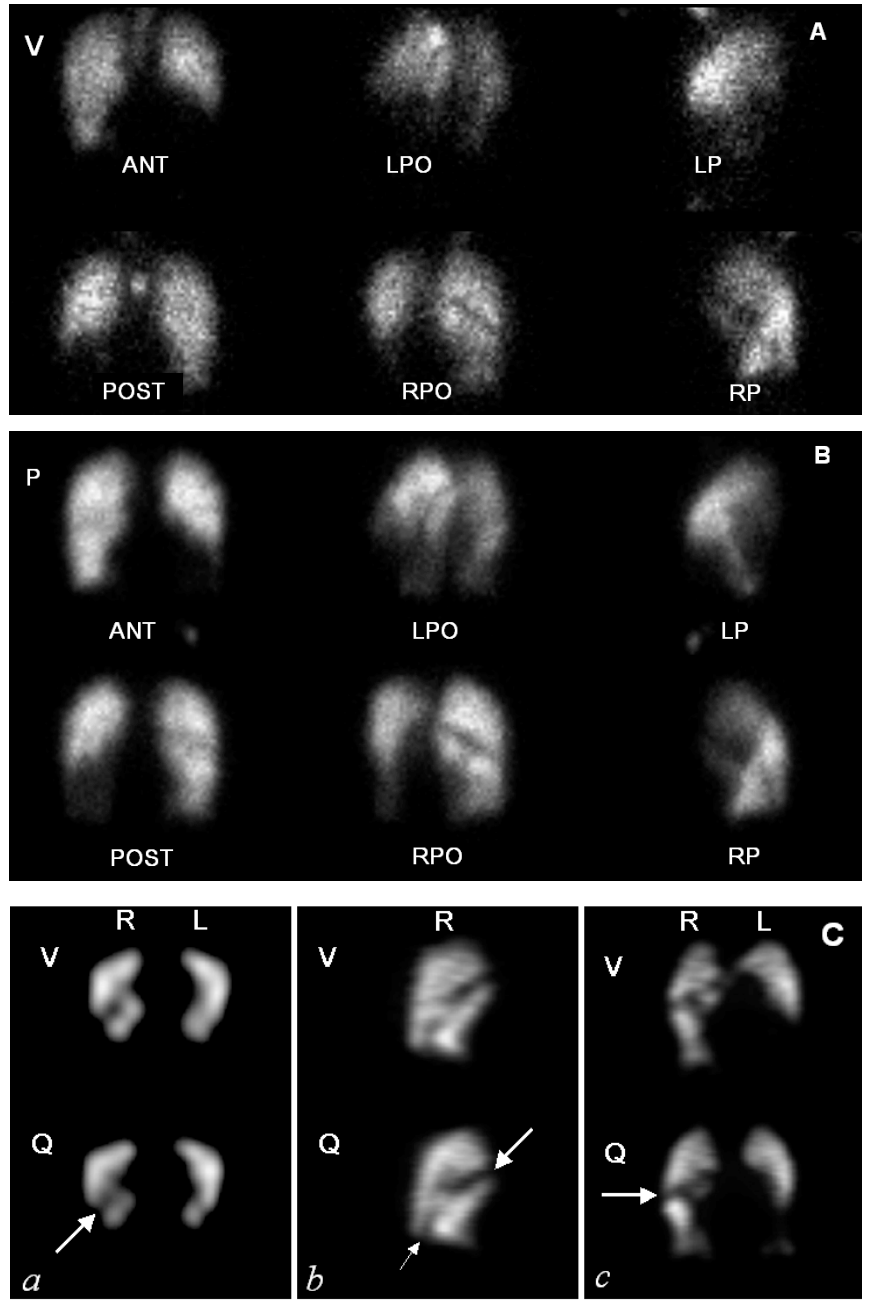

Fig. (2). Patient with no pulmonary embolism. CT scan found infiltrates in the upper lobes and bilateral pleuresia. US was negative. Perfusion defects are marked by arrows. Planar perfusion (B) and planar ventilation (A) show bilateral matched defects. Ventilation/perfusion tomography $(\mathbf{C})$ clearly shows that these defects correspond to scissures.

$\mathrm{V}=$ ventilation; $\mathrm{Q}=$ perfusion; $\mathrm{ANT}=$ anterior; $\mathrm{POST}=$ posterior; $\mathrm{RP}=$ right profile; $\mathrm{LP}=$ left profile; $\mathrm{RPO}=$ right posterior oblique; $\mathrm{LPO}=$ left posterior oblique; $\mathrm{R}=$ right; $\mathrm{L}=$ left; $\mathrm{a}=$ transaxial slices; $\mathrm{b}=$ sagittal slices of right lung; $\mathrm{c}=$ coronal slices.

and sural veins. The criterion for diagnosing deep-venous thrombosis was non-compressibility of the vein and/or the presence of a thrombus. Upper-limb US was not performed.

\section{Establishing and Excluding Pulmonary Embolism}

The diagnosis of PE was confirmed if: (1) multidetectorrow CT showed a picture of thrombus in a main or lobar or a segmental branch of a pulmonary artery or at least two thrombus at the subsegmental level and/or (2) US showed a thrombus or/and non compressibility of the vein.

The diagnosis of PE was ruled out if both multidetectorrow CT and US showed no-thrombus, except in patients with high clinical probability of PE in whom pulmonary angiography had to be performed.

In patients with single sub-segmental thrombus on CT, since specificity of such findings is low, the diagnosis of PE 
was established if one at least of the following criteria was present: a high clinical probability of PE, a positive lowerlimb US, a corresponding defect on lung SPECT.

\section{Follow-Up}

In patients without PE but a V/Q lung SPECT read as very high probability for PE, a 6-month follow-up was performed.

\section{Statistical Analysis}

Continuous variables are expressed as mean \pm SD and categoric data are expressed as percentage. True and false SPECT results were assessed on the basis of the diagnosis given by the association of multidetector-row CT and US. The $\kappa$ statistic was used as a measure of agreement between (1) the two readers of lung SPECT and (2) lung SPECT and $\mathrm{CT} / \mathrm{US}$ results. A value of 1 denotes perfect agreement, and 0 indicates no agreement beyond chance. In general, $\kappa$ values $\geq 0.5$ are considered indicative of good agreement.

\section{RESULTS}

\section{Study Population}

The tomographic images from one hundred and forty-two patients out of 392 (Fig. 3) with clinically suspected acute $\mathrm{PE}$ and a non-diagnostic V/Q lung scan were initially interpreated. Forty-five patients (18 male, 27 female, mean-age $77.1 \pm 13.2$ y) were secondarily excluded because of absolute contraindications to the administration of contrast medium (36 patients with renal failure defined by a serum creatinine level exceeding $200 \mu \mathrm{mol} / \mathrm{L}, 9$ patients with prior hypersensitivity to contrast medium).

Two patients were excluded from analysis because of unsatisfactory opacification of the pulmonary arteries.

We evaluated 95 patients (32 male, 63 female, mean-age $63.6 \mathrm{yr} \pm 18.2)$. Thirty one patients $(33 \%)$ were known to have an obstructive lung disease and 67 patients $(70.5 \%)$ had an abnormal chest-ray on admission.

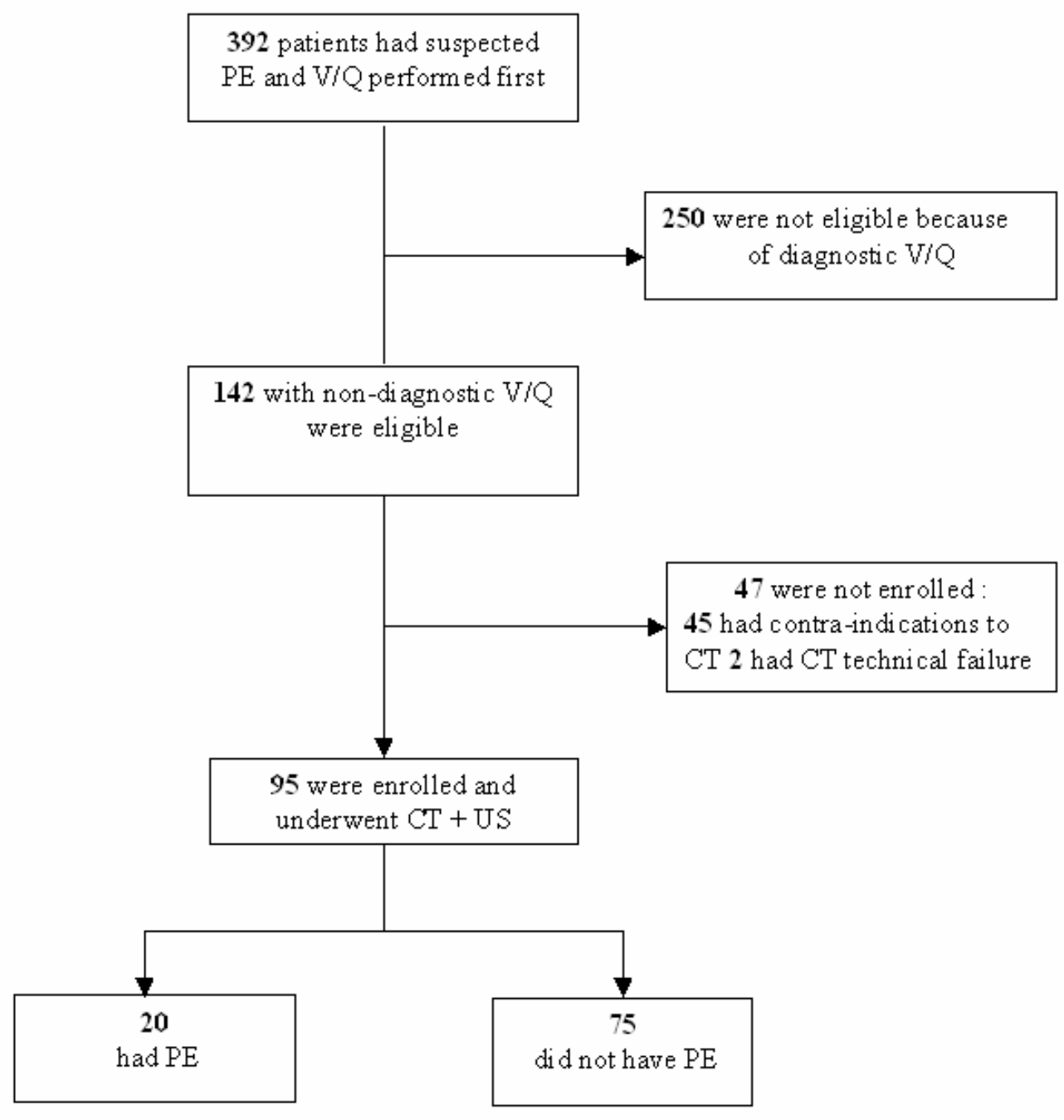

Fig. (3). Flow chart summarizing the enrollment and diagnostic procedure. 
Diagnosis of PE was done in 20 patients (21\%), 15 with a positive multidetector-row $\mathrm{CT}$ and 5 with a positive lowerlimb US. PE was ruled-out in 75 patients (79\%) with both negative CT and US. No patient had to undergo pulmonary angiography.

\section{Lung SPECT Findings}

Lung SPECT findings were concordant with final diagnosis in 77 patients (82\%), 15 patients with $\mathrm{PE}$ and 62 patients without. (Table 1)

In 17 patients (18\%), lung SPECT findings were discordant with final diagnosis, 4 patients (4\%) with PE and 13 (14\%) patients without.

Lung SPECT was non-diagnostic in 1 patient with a triple match defect corresponding to PE with pulmonary infarction.

Table 1. Concordance Between Lung SPECT and the Association of Multidetector-Row CT and US for Classification of Patients as PE or no PE

\begin{tabular}{|c|c|c|c|}
\hline & & & \\
\hline & & No PE & PE \\
\hline & No PE & $62(66 \%)$ & $4(4 \%)$ \\
\hline SPECT & PE & $13(14 \%)$ & $15(16 \%)$ \\
\hline & & $\begin{array}{l}\text { Agreemer } \\
\kappa=0.50\end{array}$ & $4(82 \%)$ \\
\hline
\end{tabular}

All 4 patients with PE and a negative lung SPECT had a single segmental non-occluding thrombus on multidetectorrow CT scan. US was performed in 3 of them and no phlebitis was found.

In 13 patients with no PE but a positive lung SPECT, all patients had sub-segmental defects only, 8 patients with a single and 5 patients with a double sub-segmental defect.

Overall diagnostic performances of V/Q SPECT are given in Table $\mathbf{2}$.

Table 2. Diagnostic Ability of Lung SPECT to Detect PE (n = 95) when V/Q Planar Lung Scan is Non-Diagnostic

\begin{tabular}{|c|c|}
\hline Parameter & Value \\
\hline \hline Sensitivity & 0.79 \\
Specificity & 0.83 \\
Negative predictive value & 0.94 \\
Positive predictive value & 0.53 \\
Accuracy & 0.80 \\
True-positive & 15 \\
True-negative & 62 \\
False-positive & 13 \\
False-negative & 4 \\
Non-diagnostic & 1 \\
$\Sigma$ & 95 \\
\hline
\end{tabular}

\section{Lung SPECT Inter-Observers Concordance}

Lung SPECT interpretation was concordant in $81(85 \%)$ patients and discordant in $14(15 \%)$ patients with $\kappa=0.60$. In discordant patients, a consensus was always reached.

\section{Follow-Up}

Follow-up was performed at 6 months in patients in whom PE was ruled out but a positive lung SPECT (13 patients). During the follow-up, two patients were lost, one patient died of cancer, one patient had a phlebitis proven by US and 9 patients were free of events. However 3 of these 9 patients were under chronic anticoagulant regimen because of atrial fibrillation at the inclusion of the study.

\section{DISCUSSION}

The goal of our study was to determine the usefulness of SPECT in patients suspected of acute PE and with a nondiagnostic V/Q lung scan. Lung tomography in combination with our developed set of criteria for image interpretation virtually eliminates non-diagnostic exams. Concordance with a reference procedure combining multidetector-row $\mathrm{CT}$ and lower- limb US is high ( $80 \%$ of patients).

Suppression of non-diagnostic scans with SPECT comes from a major increase in image quality resulting in better confidence in image interpretation and from our interpretation chart. It has been shown that overlapping structures result in image worsening when planar imaging is used [20, $21]$ and this is particularly true in lung scan where numerous segments are entangled. SPECT enables to get rid of most of the environment when analysing a specific zone and the visual comfort brought by its use is obvious. It should be reminded that SPECT has totally eclipsed the planar mode in myocardial and brain scintigraphy but V/Q lung scan is still performed in planar mode in most of institutions.

A high concordance between lung SPECT and the final diagnosis was obtained by means of specific criteria of interpretation. These criteria were developed during a previous step in which a variety of findings on SPECT exams were compared to multidetector-row CT images. These criteria result from our own experience and can be criticized but at this time they are the only ones to be available in the setting of non-diagnostic lung scan. One could argue that it is unfair to perform a comparison between planar and SPECT imaging using different criteria of interpretation. One must realise that the criteria we developed for SPECT interpretation cannot be used for planar imaging. SPECT imaging enables to assess the shape of defects, the presence of small subsegmental defects or extension of a defect into the treasure for example. This cannot be seen on planar imaging. Lung scan interpretation relies mainly on the notion of matched or mismatched defect. A mismatch defect however can be found not only in PE but also in other conditions such as vascular compression by a tumor, lung vasculitis or idiopathic pulmonary fibrosis. With a major improvement in image quality, SPECT enables to go further into the interpretation process.

Use of our criteria lead to a diagnosis in all but one patient who had a triple match defect, i.e. a chest opacity with a 
matched V/Q defect. Since this kind of finding may correspond either to pulmonary infarction (which was the right diagnosis in this case) or a parenchymal disease (such as pneumonia), lung SPECT cannot reliably differentiate these two conditions. It should be noted that no SPECT was considered as non-diagnostic because of insufficient image quality. Even though ventilation scans may exhibit non-optimal quality in patients with advanced obstructive lung diseases when using Technegas [22], this was never, in our series, a reason for technical failure. Diagnostic performances in our study should be compared with PIOPED II latest results [23] where sensitivity and specificity of V/Q scanning reached $77 \%$ and $98 \%$ respectively once nondiagnostic studies were removed. Our data show that lung SPECT exhibits an excellent negative predictive value to rule out PE when planar lung scan is non-diagnostic, which is similar to the negative predictive value of D-dimer levels less than $500 \mathrm{ng} / \mathrm{mL}$. If confirmed by others, a negative lung SPECT would safely rule out $\mathrm{PE}$ in patients with a non-diagnostic lung scan. Positive predictive value is rather disappointing but related to our diagnostic workup for PE, based upon the results of multidetector-row CT combined with US. Although such a strategy has been validated and is now widely used, it may have some shortcomings, particularly when PE is falsely ruled out. The association of CT scan and US has been validated by outcome studies showing that anticoagulation withhold is safe when PE is excluded [1-9]. This does not mean that patients with both negative CT scan and US have no PE. In Pioped II, $11 \%$ of patients with a negative CT scan and an intermediate probability clinical assessment for PE had PE [1]. However, undiagnosed small PE shows a low incidence of symptomatic recurrent PE. As recently pointed out by Perrier et al. [24] and Stein et al. [25], outcome studies and accuracy studies do not equate. As our study was an accuracy study, the lack of a real gold standard to diagnose PE should lead to a careful interpretation of the diagnostic performance of lung SPECT and a comprehensive analysis of patients with discordant results. Seventeen patients $(18 \%)$ had discordant diagnosis on lung SPECT according to our diagnosis procedure, 13 patients (14\%) without $\mathrm{PE}$ and 4 patients (4\%) with PE. All thirteen patients with no PE on CT but a positive lung SPECT had sub-segmental perfusion defects only, 8 of which had a single sub-segmental defect. These patients can be considered as false positive, i.e. a misleading interpretation of lung SPECT. Multidetectector-row CT has not however a $100 \%$ sensitivity in detecting distal sub-segmental thrombus. A six-month follow-up for thrombo-embolic events was performed in order to assess the prognosis of such findings but our small population did not lead to definite conclusions. Because therapeutic abstention in such patients seems associated with a favourable outcome [26], there is currently no mean of certainty at this time to determine whether these sub-segmental defects correspond to PE or no.

Four patients had segmental PE on CT without corresponding defect on SPECT. These four clots were considered as non-occlusive. No segmental occluding thrombus was missed by lung SPECT. Perfusion lung scan is a functional imaging modality where albumin macroaggregates are infused and trapped in the capillary bed. If a non-occlusive thrombus is present, albumin macroaggregates can still reach the capillary bed and no defect will be observed. Since per- fusion V/Q and CT scan are two imaging modalities relying on different principles, i.e. visualization of a perfusion defect versus imaging of a thrombus, this kind of discrepancy is not surprising. Whether a non-occlusive segmental thrombus has the same prognosis as an occlusive one remains to be evaluated.

Our findings do not confirm the retrospective study of Reinartz et al. [17] where lung SPECT did not prove to be superior to planar mode in patients with a non-diagnostic lung scan. Major differences in methodology such as a questionable diagnostic work-up for PE [27], inclusion criteria and SPECT interpretation criteria probably account for this discrepancy. If lung SPECT could not help in patients with non-diagnostic planar lung scan and could only increase the number of detectable defects as well as change the established value of a normal planar lung scan, lung SPECT would offer a dubious clinical help.

In our study, the proportion of patients in whom CT scan was contra-indicated (31\%) is somewhat high but illustrates the daily routine. Because of the design of our study, only patients with non-diagnostic planar V/Q lung scan had been analysed. Most of these patients had an abnormal chest radiography as well as an underlying lung and/or heart disease. Patients not eligible for CT were older. These population characteristics explain the high proportion of contraindication to CT. Although new generation CT minimize indepth radiation exposure, the absorbed dose to the breast is however at best not diminished and remains a concern. The high proportion of patient drop-out for CT on one hand and radiation burden on the other, stress the need for a technique with no or few contra-indications and side-effects.

In daily practice, V/Q lung scan is performed as a firstline imaging test in patients with normal X-ray only or when CT scan is contra-indicated. In many institutions, CT scan is performed as a first-line imaging test in all patients. Our study demonstrates that lung SPECT can dramatically lower the rate of non-diagnostic scans and that overall agreement with CT scan is high. One could argue that V/Q scan should be performed as a first-line imaging test only in patients with contra-indications to CT scan. This may represent a very significant proportion of patients in routine, as high as $40 \%$ [28 ]. Since most patients who undergo a CT scan do not have $\mathrm{PE}$, alternate imaging modalities without side-effects should be promoted. In patients with chest radiography opacities who need further diagnostic procedures, CT scan is justified as a first-line imaging test since it may bring an alternate diagnosis to PE. In patients with abnormal X-ray because of obstructive lung disease or heart congestion in whom V/Q scan is generally non-diagnostic, the use of lung SPECT should lead to a renewed interest. In these patients, since V/Q lung SPECT has no contra-indications and no side-effects, its use as a first-line diagnostic imaging test seems justified. Lung SPECT did perform reliably with no technical failure and good inter-observers reproducibility. Software under development and respiratory gating [29, 30] will probably help in routine to rapidly assess regions of mismatch and to improve overall diagnostic performances.

\section{CONCLUSION}

Our study shows that nearly all non-diagnostic V/Q lung scans $(99 \%)$ can become diagnostic when SPECT is per- 
formed. Correlation with a procedure combining multidetector-row CT scan and lower-limb US when using our criteria of interpretation is high in patients referred for suspicion of acute PE. Discordance was found only in patients with subsegmental defects on lung SPECT or non-occluding segmental thrombus on multi-slices CT. Lung SPECT exhibits an excellent negative predictive value to rule out PE. Easy to perform on a daily basis, with no contra-indications and no side-effects, the performance of lung SPECT should lead to position lung tomography as a useful tool in the diagnosis strategy of PE and relegate the planar mode.

\section{ACKNOWLEDGEMENTS}

The authors wish to thank Pr. Serge Askienazy, Dr. Georges Baillet, Dr. Jean-Marie Rocchisani and Dr. Yoann Lalatonne for their help.

Supported by a grant from Direction de la Recherche Clinique, APHP, Paris, France.

\section{REFERENCES}

[1] Stein PD, Woodard PK, Weg JG, et al. Diagnostic pathways in acute pulmonary embolism: recommendations of the PIOPED II investigators. Am J Med 2006; 119: 1048-1055.

[2] Kruip MJ, Leclercq MG, van der Heul C, et al. Diagnostic strategies for excluding pulmonary embolism in clinical outcome studies: a systematic review. Ann Intern Med 2003; 138: 941-51.

[3] Goldhaber SZ. Pulmonary embolism. Lancet 2004; 363: 1295-305.

[4] Lorut C, Ghossains M, Horellou MH, et al. A Noninvasive diagnostic Strategy Including Spiral Computed Tomography in Patients with Suspected Pulmonary Embolism. Am J Respir Crit Care Med 2000; 162: 1413-1418.

[5] Musset D, Parent F, Meyer G, et al. Diagnostic Strategy for patients with suspected pulmonary embolism: a prospective multicenter outcome study. Lancet 2002; 360: 1914-20.

[6] Perrier A, Roy PM, Aujesky D, et al. Diagnosing pulmonary embolism in outpatients with clinical assessment, D-dimer measurement, venous ultrasound, and helical computed tomography: a multicenter management study. Am J Med 2004; 116: 291-9.

[7] Van Strijen MJL, de Monye W, Kieft GJ, et al. Accuracy of singledetector spiral $\mathrm{CT}$ in the diagnosis of pulmonary embolism: a prospective multi-center cohort study of consecutive patients with abnormal perfusion scintigraphy. J Thromb Haemost 2005; 3: 17-25. Erratum in J Thromb Haemost 2005; 3: 622.

[8] Perrier A, Roy PM, Sanchez O, et al. Multidetector-Row Computed Tomography in Suspected Pulmonary Embolism. N Engl Med 2005; 352: 1760-8.

[9] Stein PD, Fowler SE, Goodman LR, et al. Multidetector computed tomography for acute pulmonary embolism. N Engl J Med 2006; 354: 2317-27.

[10] Parker MS, Hui FK, Camacho MA, et al. Female breast radiation exposure during CT pulmonary angiography. Am J Roentgenol 2005; 185: 1228-1233.

[11] Cook JV, Kyriou J. Radiation from CT and perfusion scanning in pregnancy. Brit Med J 2005; 331: 350.
[12] International commission on radiological protection. ICRP publication 87: managing patient dose in computed tomography. Ann ICRP 2000; 30: 7-45.

[13] Kalra MK, Maher MM, Saini S. CT radiation exposure: rationale for concern and strategies for dose reduction. Proceedings from the SCBT/MR. Applied Radiol 2003; 7: 45-54

[14] Corbus HF, Seitz JP, Larson RK, et al. Diagnostic usefulness of lung SPET in pulmonary thromboembolism: an outcome study. Nucl Med Commun 1997; 18: 897-907.

[15] Palmer J, Bitzen U, Jonson B, et al. Comprehensive Ventilation/Perfusion SPECT. J Nucl Med 2001; 42: 1288-1294.

[16] Bajc M, Bitzen U, Olsson B, et al. Lung Ventilation/Perfusion SPECT in the Artificially Embolized Pig. J Nucl Med 2002; 43 : 640-647.

[17] Reinartz P, Wildberger J, Schaefer W, et al. Tomographic Imaging in the Diagnosis of Pulmonary Embolism: A Comparison Between V/Q lung Scintigraphy in SPECT Technique and Multislice Spiral CT. J Nucl Med 2004; 45: 1501-1508.

[18] Bajc M, Olsson CG, Palmer J, et al. Diagnostic evaluation of planar and tomographic ventilation/perfusion lung images in patients with suspected pulmonary emboli. Clin Physiol Funct Imaging 2004; 24: 249-56.

[19] Harris B, Schembri G, Bailey D, et al. A comparison of planar V/Q, SPECT derived planar reconstruction and SPECT scintigraphy in the diagnosis of pulmonary embolism. [abstract ] J Nucl Med 2006; 47 (suppl 1) 357 P.

[20] Morrell NW, Nijran KS, Jones BE, et al. The underestimation of segmental defect size in radionuclide lung scanning. J Nucl Med 1993; 34: 370-374.

[21] Magnussen JS, Chicco P, Palmer AW, et al. Single-photon emission tomography of a computerised model of pulmonary embolism. Eur J Nucl Med 1999; 26: 1430-1438.

[22] Howart MD, Lan L, Thomas AP, et al. Tc Technegas ventilation and perfusion lung scintigraphy for the diagnosis of pulmonary embolus. J Nucl Med 1999; 40: 579-584.

[23] Sostman HD, Stein PD, Gottschalk A, et al. Acute pulmonary embolism: sensitivity and specificity of ventilation-perfusion scintigraphy in PIOPED II study. Radiology 2008; 246: 941-46.

[24] Perrier A, Bounameaux H. Accuracy or outcome in suspected pulmonary embolism. N Engl J Med 2006; 354: 2383-2384.

[25] Stein PD, Beemath A, Goodman LR, et al. Outcome studies of pulmonary embolism versus accuracy: they do not equate. Thromb Haemost 2006; 96: 107-108

[26] Le Gal G, Righini M, Parent F, et al. Diagnosis and management of subsegmental pulmonary embolism. J Thromb Haemost 2006; 4: 724-731.

[27] De Geeter FW. Tomographic imaging in the diagnosis of pulmonary embolism: still, we do not know. J Nucl Med 2005; 46: 2119 2121.

[28] Colignon MA, Benada A, Meyer G. Pulmonary embolism diagnosis: will lung scintigraphy survive ? [abstract] J Nucl Med 2002; (suppl): 162P.

[29] Suga K, Kawakami Y, Zaki M, et al. Assessment of regional lung functional impairment with co-registered respiratory-gated ventilation/perfusion SPECT-CT images: initial experiences. Eur J Nucl Med Mol Imaging 2004; 31: 240-49.

[30] Harris B, Bailey D, Miles S, et al. Objective analysis of tomographic ventilation-perfusion scintigraphy in pulmonary embolism. Am J Respir Crit Care Med 2007; 175: 1173-1180. 